\title{
Modulation of Chloride Channel Functions by the Plant Lignan Compounds Kobusin and Eudesmin
}

\author{
Yu Jiang ${ }^{1 \dagger}$, Bo Yu ${ }^{1+}$, Fang Fang ${ }^{1}$, Huanhuan $\mathrm{CaO}^{1}$, Tonghui Ma ${ }^{2}$ and Hong Yang ${ }^{1 *}$ \\ ${ }^{1}$ School of Life Sciences, Liaoning Provincial Key Laboratory of Biotechnology and Drug Discovery, Liaoning Normal \\ University, Dalian, China, ${ }^{2}$ College of Basic Medical Sciences, Dalian Medical University, Dalian, China
}

\section{OPEN ACCESS}

Edited by:

Domenico De Martinis, ENEA Italian National Agency for New Technologies, Energy and Sustainable Economic Development, Italy

Reviewed by:

Loretta Ferrera,

U.O.C. Genetica Medica- I. G. Gaslini,

Anna Boccaccio

National Research Council, Italy

*Correspondence:

Hong Yang

hyangInnu@126.com

${ }^{t}$ These authors have contributed equally to this work.

Specialty section: This article was submitted to Plant Biotechnology, a section of the journal

Frontiers in Plant Science

Received: 09 September 2015 Accepted: 09 November 2015 Published: 25 November 2015

Citation:

Jiang Y, Yu B, Fang F, Cao H, Ma T and Yang $H$ (2015) Modulation of Chloride Channel Functions by the Plant Lignan Compounds Kobusin and Eudesmin. Front. Plant Sci. 6:1041. doi: $10.3389 /$ fpls.2015.01041
Plant lignans are diphenolic compounds widely present in vegetables, fruits, and grains. These compounds have been demonstrated to have protective effect against cancer, hypertension and diabetes. In the present study, we showed that two lignan compounds, kobusin and eudesmin, isolated from Magnoliae Flos, could modulate intestinal chloride transport mediated by cystic fibrosis transmembrane conductance regulator (CFTR) and calcium-activated chloride channels (CaCCs). The compounds activated CFTR channel function in both FRT cells and in HT-29 cells. The modulating effects of kobusin and eudesmin on the activity of $\mathrm{CaCC}_{\text {gie }}(\mathrm{CaCC}$ expressed in gastrointestinal epithelial cells) were also investigated, and the result showed that

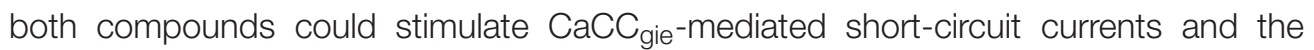
stimulation was synergistic with ATP. In ex vivo studies, both compounds activated CFTR and $\mathrm{CaCC}_{\text {gie }}$ chloride channel activities in mouse colonic epithelia. Remarkably, the compounds showed inhibitory effects toward ANO1/CaCC-mediated short-circuit currents in ANO1/CaCC-expressing FRT cells, with $\mathrm{IC}_{50}$ values of $100 \mu \mathrm{M}$ for kobusin and $200 \mu \mathrm{M}$ for eudesmin. In charcoal transit study, both compounds mildly reduced gastrointestinal motility in mice. Taken together, these results revealed a new kind of activity displayed by the lignan compounds, one that is concerned with the modulation of chloride channel function.

\section{Keywords: CFTR, CaCCs, ANO1/CaCC, kobusin, eudesmin, short-circuit current}

\section{INTRODUCTION}

Plant lignans are widely distributed in vegetables, fruits, and grains, especially in rye, flax, and sesame seeds. It has been reported that lignans have various biological activities, including anti-cancer, anti-diabetic, antimicrobial, antiparasitic, and antihypertensive activities (Dar and Arumugam, 2013; Chun et al., 2014; Zhang et al., 2014a). However, little information is available on lignan compounds and chloride channels.

Active $\mathrm{Cl}^{-}$secretion mediated by chloride channels provides a driving force for the transepithelial fluid secretion in the apical membrane of the intestines. It has been fully established that cystic fibrosis (CF) transmembrane conductance regulator (CFTR) and calciumactivated chloride channels ( $\mathrm{CaCCs}$ ) are the main chloride channels present in the luminal membrane of enterocytes (Riordan et al., 1989; Hartzell et al., 2005). CFTR is a cAMPdependent chloride channel predominantly expressed in the crypt cells in the intestines, and is permeable to $\mathrm{Cl}^{-}$and $\mathrm{HCO}_{3}^{-}$(Zhang et al., 2012). ANO1/CaCC (TMEM16A) is the 
first molecular identity of CaCCs that was found to express abundantly in the intestinal pacemaker Cajal cells, where it generates smooth muscle contraction (Huang et al., 2009; Hwang et al., 2009; Ferrera et al., 2010). CaCC gie (CaCC that located in the gastrointestinal epithelial cells), which is $\mathrm{CaCC}$ apart from ANO1, is predominantly localized in the gastrointestinal epithelial cells and is involved in fluid secretion, though its molecular identity remains unclear.

Hyper activation of CFTR and CaCC proteins may account for such diseases as secretory diarrhea (Morris et al., 1999) and autosomal dominant polycystic kidney disease (Li et al., 2004), while dysfunction of these proteins may lead to CF (Kerem et al., 1989; Riordan et al., 1989), chronic pancreatitis (Cohn, 2005) as well as constipation (Morris et al., 1999). Chronic constipation (CC) is a common symptom characterized by infrequent stools and/or difficult stool passage (Lembo and Camilleri, 2003). The etiology of constipation is very complicated and may include diet, impaired colonic motility, behavioral and psychological factors (Lu et al., 2015). Current treatments are mainly based on dietary management and the use of laxatives, which usually show discouraging results. Therefore there is an urge to find new strategies for CC therapy. During the last decade, emphasis has been placed on increasing the intestinal fluid secretion and gastrointestinal motility as a new therapeutic option for the treatment of CC.

Previously, we have set up a high throughput screening strategy for identifying natural active compounds against chloride channels (Zhang et al., 2014b; Chen et al., 2015). Based on this strategy, we found a large number of compounds, including two lignan compounds, kobusin and eudesmin, which had CFTR and $\mathrm{CaCC} \mathrm{Cl}^{-}$channel modulation activities. The aim of the present study was to systematically investigate the modulation effects of kobusin and eudesmin on CaCCs and CFTR chloride channel activities. We demonstrated for the first time that plant lignan compounds could modulate intestinal chloride transport mediated by CFTR and CaCCs chloride channels.

\section{MATERIALS AND METHODS}

\section{Cell Lines, Animals, and Compounds}

Cell lines used in this study were FRT (fischer rat thyroid epithelial) cells stably co-transfected with the YFP-H148Q fluorescence protein and human wild-type CFTR cDNA (Clarke et al., 2001; Harmon et al., 2010) or ANO1 cDNA (Hao et al., 2011) and HT-29 cells. FRT cells were cultured in Nutrient F12 coon's medium (Sigma Chemical Co. St. Louis, MO, USA). HT29 cells were cultured in 1640 medium (Sigma Chemical Co. St. Louis, MO, USA). Both media were supplemented with $10 \%$ fetal bovine serum (HyClone company, USA), $100 \mathrm{u} / \mathrm{ml}$ penicillin, $100 \mu \mathrm{g} / \mathrm{ml}$ streptomycin and $2 \mathrm{mM} \mathrm{L}$-glutamine. The cells were incubated in a $5 \% \mathrm{CO}_{2}$ incubator maintained at $37^{\circ} \mathrm{C}$ and $95 \%$ humidity before they were used for iodide influx fluorescence study and short-circuit current measurement.

Male ICR mice (8-10 weeks) were fed a standard chow diet and kept under specific pathogen-free conditions at Dalian
Medical University (Permit Number: SCXK liao 2008-0002). All animal experiments were conducted in accordance with the Guide for the Care and Use of Laboratory Animals of the National Institutes of Health and were approved by the Liaoning Normal University Committee on Animal Research.

CFTR $_{\text {inh }}-172$ was synthesized as described previously (Garcia et al., 2009). Forskolin (FSK), genistein (Gen), indomethacin, amiloride and tannic acid were all purchased from Sigma (Sigma Chemical Co, St. Louis, MO, USA). Amphotericin B was purchased from Solarbio (Beijing Solarbio Science \& Technology Co, Ltd). $\mathrm{CaCC}_{\text {inh }}$-A01 and $\mathrm{E}_{\text {act }}$ were obtained from Chembest Research Laboratory Limited (Shanghai). ATP and NaI were purchased from Sangon Biotech (Shanghai) Co, Ltd. Kobusin and eudesmin were isolated and purified in our own laboratory and their chemical structures are shown in Figure 1.

\section{lodide Influx Fluorescent Assay}

FRT cells transfected with CFTR were plated in a black 96well plate with clear bottom (Costar, Corning, NY, USA) at a density of $2 \times 10^{4}$ cells/well and incubated until confluent. The cells were washed three times with PBS followed by the addition of FSK (100 nM per well) and a further incubation of $5 \mathrm{~min}$. After that, kobusin or eudesmin was added to each well at different concentrations and the cells were incubated for another $15 \mathrm{~min}$. YFP fluorescence data were recorded using a FLUOstar Galaxy microplate reader (BMG Lab Technologies, Inc.) equipped with HQ500/20X (500 $\pm 10 \mathrm{~nm})$ excitation, HQ 535/30M (535 $\pm 15 \mathrm{~nm}$ ) emission filters (Chroma Technology Corp.) and syringe pumps. Iodide influx rates $(\mathrm{d}[\mathrm{I}-\mathrm{d} / \mathrm{dt})$ were computed as described by Kristidis et al. (1992).

\section{Short-circuit Current}

Snapwell inserts containing ANO1-expressing FRT cells and HT-29 cells were mounted in Ussing chambers (Physiological Instruments, San Diego, CA, USA). For FRT cells, the hemichambers were filled with $5 \mathrm{ml}$ of half- $\mathrm{Cl}^{-}$solution (apical) and $\mathrm{HCO}_{3}{ }^{-}$buffered solution (basolateral). The half- $\mathrm{Cl}^{-}$solution contained $65 \mathrm{mM} \mathrm{NaCl}, 65 \mathrm{mM} \mathrm{Na}$ Gluconate, $2.7 \mathrm{mM} \mathrm{KCl}$, $1.5 \mathrm{mM} \mathrm{KH}_{2} \mathrm{PO}_{4}, 0.5 \mathrm{mM} \mathrm{MgCl}, 2 \mathrm{mM} \mathrm{CaCl}_{2}, 10 \mathrm{mM}$ Hepes, $10 \mathrm{mM}$ Glucose, and $25 \mathrm{mM} \mathrm{NaHCO}_{3}$ at $\mathrm{pH}$ 7.4. The $\mathrm{HCO}_{3}{ }^{-}$ buffered solution contained $120 \mathrm{mM} \mathrm{NaCl}, 5 \mathrm{mM} \mathrm{KCl}, 1 \mathrm{mM}$ $\mathrm{MgCl}_{2}, 1 \mathrm{mM} \mathrm{CaCl}, 10 \mathrm{mM}$ glucose, $5 \mathrm{mM}$ Hepes, and $25 \mathrm{mM} \mathrm{NaHCO}_{3}$ at $\mathrm{pH}$ 7.4. Snapwell inserts were mounted in Ussing Chamber systems, with the resistance kept above $1500 \Omega$. Basolateral membrane was permeabilized with amphotericin B $(250 \mu \mathrm{g} / \mathrm{ml})$. For HT-29 cells, the Krebs' buffered solution

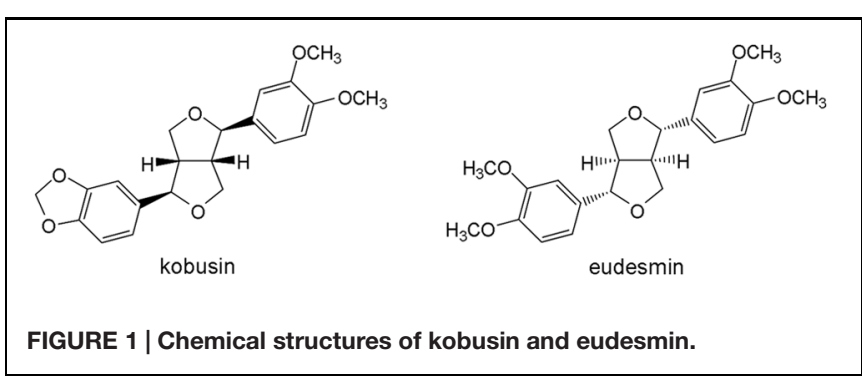


contained $130 \mathrm{mM} \mathrm{NaCl}, 2.7 \mathrm{KCl}, 1.5 \mathrm{mM} \mathrm{KH}_{2} \mathrm{PO}_{4}, 0.5 \mathrm{mM}$ $\mathrm{MgCl}_{2}, 2 \mathrm{mM} \mathrm{CaCl}$, $10 \mathrm{mM}$ Hepes, $10 \mathrm{mM}$ glucose at $\mathrm{pH}$ 7.4. Symmetrical $\mathrm{HCO}_{3}{ }^{-}$buffered solutions contained $119 \mathrm{mM} \mathrm{NaCl}$, $0.6 \mathrm{mM} \mathrm{KH}_{2} \mathrm{PO}_{4}, 2.4 \mathrm{mM} \mathrm{K} \mathrm{HPO}_{4}, 1.2 \mathrm{mM} \mathrm{MgCl}_{2}, 1.2 \mathrm{mM}$ $\mathrm{CaCl}_{2}, 21 \mathrm{mM} \mathrm{NaCO}_{3}, 10 \mathrm{mM}$ glucose at $\mathrm{pH}$ 7.4. The cells were bathed in the buffered solution for $15 \mathrm{~min}$ at $37^{\circ} \mathrm{C}$ in the presence of $95 \% \mathrm{O}_{2} / 5 \% \mathrm{CO}_{2}$

Male ICR mice were sacrificed by an overdose of intraperitoneal sodium pentobarbital. The colon was removed as quickly as possible and washed with ice-cold modified Krebs-bicarbonate solution containing $120 \mathrm{mM} \mathrm{NaCl}, 5 \mathrm{mM}$ $\mathrm{KCl}, 1 \mathrm{mM} \mathrm{MgCl} 2,1 \mathrm{mM} \mathrm{CaCl}, 10 \mathrm{mM}$ D-glucose, $5 \mathrm{mM}$ Hepes, and $25 \mathrm{mM} \mathrm{NaHCO}_{3}$ at $\mathrm{pH}$ 7.4. After stripping of the muscularis, the tissue was mounted in an Ussing Chamber system (Physiological Instruments) connected to a VCC MC 6 multi-channel voltage-current clamp via silver/AgCl electrodes and $3 \mathrm{M} \mathrm{KCl} \mathrm{Ag}$ bridges. The hemi-chambers were separately filled with $5 \mathrm{ml}$ modified Krebs-bicarbonate solution bubbled with $95 \% \mathrm{O}_{2} / 5 \% \mathrm{CO}_{2}$ at $37^{\circ} \mathrm{C}$. The hemi-chambers were filled with buffer solution containing $10 \mu \mathrm{M}$ indomethacin to prevent the influence of prostaglandin, and the mucosal side of the tissue was exposed to $10 \mu \mathrm{M}$ amiloride to inhibit epithelial $\mathrm{Na}^{+}$ current. Short-circuit current was recorded using Acquire and Analyze 2.3 software, with the transepithelial potential clamped at $0 \mathrm{mV}$ during the whole experiment.

\section{Intestinal Motility Measurement}

ICR mice were starved for $24 \mathrm{~h}$ and the animals were then orally administered PBS, $400 \mu \mathrm{M}$ kobusin or eudesmin. Fifteen minutes later, the animals were administered $200 \mu \mathrm{l}$ of $10 \%$ activated charcoal diluted in 5\% gum Arabic. Thirty minutes after the administering of activated charcoal the animals were sacrificed and the small intestines were removed. Peristaltic index was calculated as the ratio of the length that activated charcoal traveled to the total length of the small intestine.

\section{Statistical Analysis}

All data were expressed as mean \pm SE or as representative traces. Student's $t$-test was used to compare test and control values, and statistical significances were considered at the $P<0.05$ level.

\section{Ethics Statement}

This study was carried out in accordance with the recommendations of "Guide for the Care and Use of Laboratory Animals of the National Institutes of Health" and were approved by the Liaoning Normal University Committee on Animal Research. All surgery was performed under sodium pentobarbital anesthesia, and possible efforts were made to minimize suffering.

\section{RESULTS}

\section{Activation of CFTR $\mathrm{Cl}^{-}$Channel Activity by Kobusin and Eudesmin}

Activation effect of kobusin or eudesmin on CFTR chloride channel activities were tested using a cell-based fluorescence assay using FRT cells transfected with human CFTR cDNA (Ma et al., 2002). A known CFTR activator Gen (Hwang et al., 1997) was used as a positive control. FSK (100 nM) was added to the cells to acquire a basal level of cAMP (Figures 2A,B). Kobusin and eudesmin activated CFTR chloride channel activity in a dose-dependent manner with $\mathrm{EC}_{50}$ values of 30 and $50 \mu \mathrm{M}$, respectively, for kobusin and eudesmin (Figure 2C). Further experiments showed that the activation effect of these compounds could be inhibited by gradient concentrations of the known CFTR inhibitor CFTR $_{\text {inh }}-172$ (Figure 2D). CFTR activation can be achieved by direct interaction with CFTR protein or activation of upstream cAMP-dependent PKA signaling pathway (Hwang and Sheppard, 1999; Schultz et al., 1999; Sheppard and Welsh, 1999). To investigate the mechanisms involved in the activation, we measured the activities of kobusin and eudesmin under different FSK concentrations. Kobusin was effective at inducing CFTR-mediated iodide influx in the absence of FSK, although the potency was relatively weaker than that in the presence of FSK (Figure 2E). On the other hand, activation of CFTR by eudesmin depended on CAMP level and phosphorylation level of CFTR more than kobusin, which is that eudesmin showed a stronger activation effect under high concentrations of FSK (Figure 2F). The results suggested that eudesmin's efficacy is more dependent on the phosphorylation level of CFTR than kobusin.

\section{Activation of CFTR Chloride Channel Activities by Kobusin and Eudesmin in HT-29 Cells}

CFTR and CaCCs are endogenously expressed in HT-29 cells (Morris and Frizzell, 1993), and therefore, short-circuit current experiment was performed to investigate the kobusin- and eudesmin-induced activation effect on CFTR chloride channel activity in HT-29 cells. All tests were done in the presence of $30 \mu \mathrm{M}$ of the CaCC-specific inhibitor $\mathrm{CaCC}_{\text {inh-A01 (De } \mathrm{La}}$ Fuente et al., 2008) to eliminate the influence of endogenous CaCC current. Figure 3A shows that both kobusin and eudesmin alone could increase the CFTR-mediated short-circuit currents in a dose-dependent manner. Kobusin and eudesmin both elicited more potent CFTR-mediated short-circuit currents in the presence of $100 \mathrm{nM}$ FSK (Figure 3B). Summarized data are shown in Figures 3A,B (down panels).

\section{Potentiation of $\mathrm{CaCC}$ Chloride Channel Activity by Kobusin and Eudesmin in HT-29 Cells}

As $\mathrm{CaCC}_{\text {gie }}$ is endogenously expressed in HT-29 cells (Morris and Frizzell, 1993), we wanted to know what effect kobusin and eudesmin would exert on this kind of chloride channel. Figure 4A indicates that both kobusin and eudesmin could activate short-circuit current, and the activation effect could be abolished by the known non-specific CFTR and CaCC inhibitor tannic acid $(100 \mu \mathrm{M})$. After pretreatment with $\mathrm{CFTR}_{\text {inh }}-172$ $(20 \mu \mathrm{M})$, kobusin and eudesmin further activated the shortcircuit current, and the activation effect was inhibited by the specific $\mathrm{CaCC}$ inhibitor $\mathrm{CaCC}_{\text {inh- }} \mathrm{A} 01$ (Figure 4B), suggesting 
A

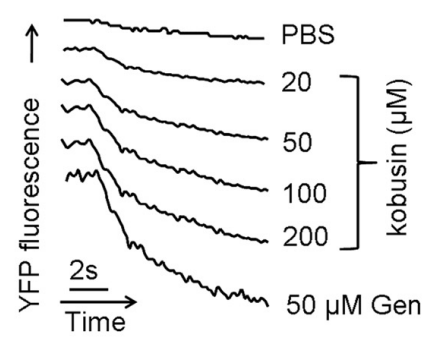

C

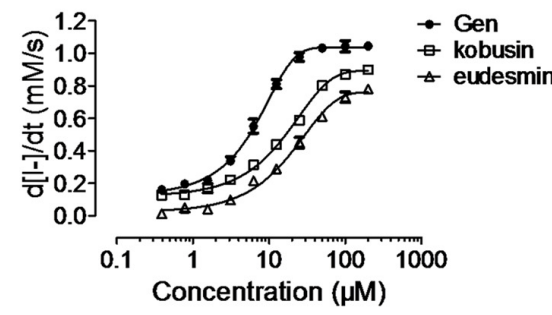

E

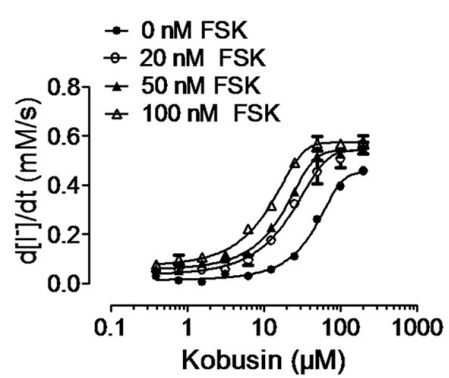

B

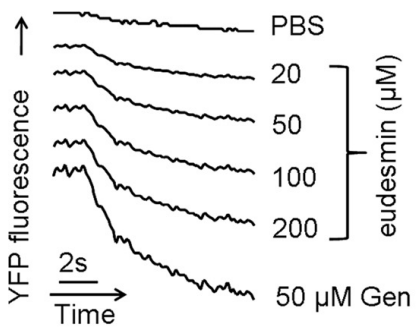

D

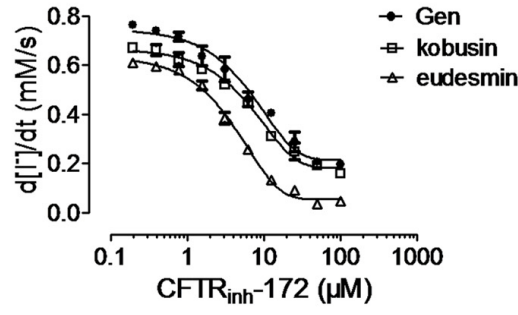

$\mathbf{F}$

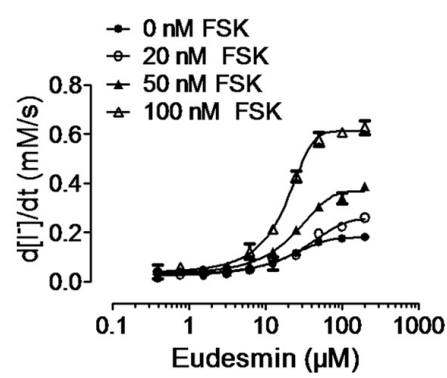

FIGURE 2 | Fluorescence quenching assay showing the activation of conductance regulator (CFTR) chloride channel activity by kobusin and eudesmin in transfected FRT cells. (A) Original traces showing quenching of YFP fluorescence by I $^{-}$addition with PBS and after additions of indicated concentrations of kobusin and Gen $(50 \mu \mathrm{M})$. (B) Original traces showing quenching of YFP fluorescence by $\mathrm{I}^{-}$addition with PBS and after additions of indicated concentrations of eudesmin and Gen $(50 \mu \mathrm{M})$. (C) Dose-dependent activation of CFTR chloride channel activity by kobusin and eudesmin. (D) Activation of CFTR $\mathrm{Cl}^{-}$channel activity by kobusin and eudesmin in the presence of gradient concentrations of CFTR inhibitor CFTR inh $^{-172}$. Dose-response effects of kobusin (E) and eudesmin $(\mathbf{F})$ on the activation of CFTR in the presence of different concentrations of FSK. Data are the means \pm SEs of three independent tests.

that both compounds were able to activate $\mathrm{CaCC}$-mediated $\mathrm{Cl}^{-}$ current.

To evaluate whether the two lignan compounds activated $\mathrm{CaCC}_{\text {gie }}$ the way ATP does, we measured the activation effects of the lignan compounds added before and after the addition of ATP. Kobusin and eudesmin induced a higher short-circuit current than DMSO (which served as a control) regardless of whether the compound was added before or after the addition of ATP (Figure 5). Kobusin $(50 \mu \mathrm{M})$ induced a higher short-circuit current than $50 \mu \mathrm{M}$ DMSO (which served as a control) and achieved a short-circuit current increase of 2.6-folds at $50 \mu \mathrm{M}$ when kobusin was added before addition of ATP (Figure 5A). When added after addition of ATP, kobusin achieved a shortcircuit current increase of 3.7-folds at $50 \mu \mathrm{M}$ (Figure 5B). Similar to kobusin, eudesmin achieved a short-circuit current increase of 2.4-folds and at $50 \mu \mathrm{M}$ when added before addition of
ATP (Figure 5C). When added after addition of ATP, eudesmin achieved a short-circuit current increase of 2.15 -folds at $50 \mu \mathrm{M}$ (Figure 5D). The results thus indicated the presence of synergistic effect between kobusin or eudesmin and ATP.

\section{Inhibitory Effects of ANO1 Chloride Channel Activities by Kobusin and} Eudesmin in ANO1-expressing FRT Cells

ANO1 is the first identified molecular component of CaCCs, and thus we investigated the effects of kobusin and eudesmin

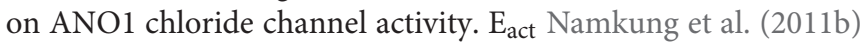
was used to produce an ANO1-mediated short-circuit current, followed by indicated concentrations of kobusin or eudesmin additions. The remaining currents were abolished by $\mathrm{T} 16 \mathrm{~A}_{\mathrm{inh}}{ }^{-}$ A01 (Namkung et al., 2011a). The results showed that apical 

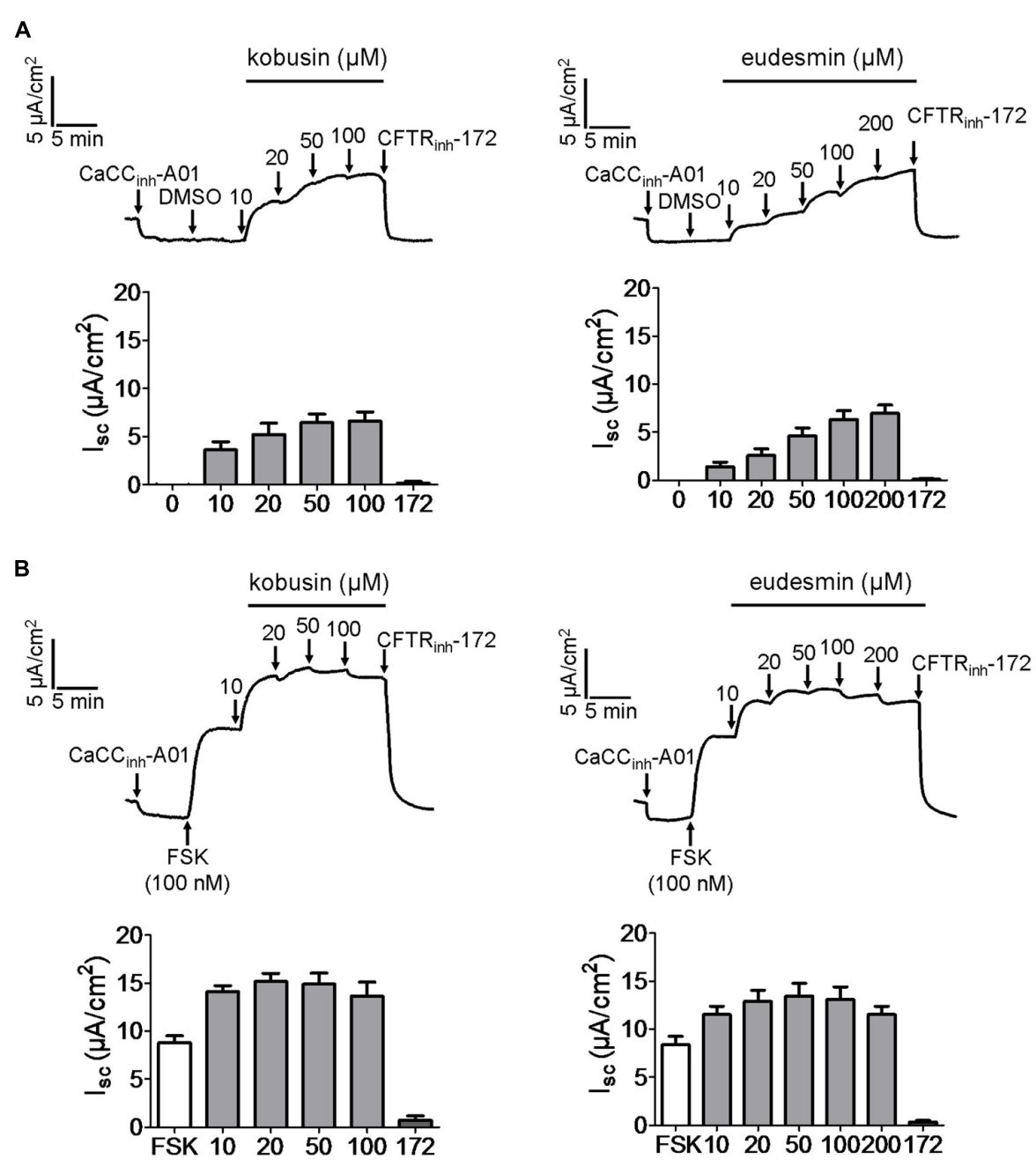

FIGURE 3 | Activation of CFTR chloride channel activities by kobusin and eudesmin in HT-29 cells. (A) Activation effect of CFTR chloride channel activity by kobusin and eudesmin without FSK. (B) Activation of CFTR chloride channel activity by kobusin and eudesmin in the presence of 100 nM FSK. CaCC inh-A01 $(30 \mu \mathrm{M})$, DMSO (100 nM) and CFTR inh $-172(50 \mu \mathrm{M})$ were added where indicated. Histograms showing summary of short-circuit currents induced by kobusin and eudesmin. 172: CFTR inh-172. Data are the means \pm SEs of three independent tests.

application of kobusin and eudesmin inhibited $\mathrm{E}_{\text {act }}$-induced ANO1-mediated short-circuit currents in transfected FRT cells in a dose-dependent manner with $\mathrm{IC}_{50}$ values of $100 \mu \mathrm{M}$ for kobusin (Figure 6A) and $200 \mu \mathrm{M}$ for eudesmin (Figure 6B). Statistical analysis is shown in Figures $6 \mathbf{A}, \mathbf{B}$ (down panels). $\mathrm{E}_{\mathrm{act}}{ }^{-}$ induced ANO1-mediated short-circuit current was completely abolished by the specific inhibitor of ANO1 T16A $\mathrm{inh}-\mathrm{A} 01$ (Figure 6C).

\section{Activation of CFTR and CaCC Chloride Channel Activities by Kobusin and Eudesmin in Mouse Colonic Epithelia}

As CFTR and CaCCs are the major pathway for apical $\mathrm{Cl}^{-}$ exit in the intestine, the efficacies of kobusin and eudesmin were tested ex vivo in isolated mouse colonic mucosa by shortcircuit current analysis. The experiments were performed in the presence of $10 \mu \mathrm{M}$ indomethacin and $10 \mu \mathrm{M}$ amiloride to eliminate the influence of prostaglandin generation and $\mathrm{Na}^{+}$ transport. Kobusin and eudesmin increased the short-circuit currents in a dose-dependent manner in mouse colonic epithelia (Figures 7A,B). As expected, the activation effect was completely abolished by $100 \mu \mathrm{M} \mathrm{CFTR}_{\text {inh }}-172$ plus $100 \mu \mathrm{M} \mathrm{CaCC}_{\text {inh }}-\mathrm{A} 01$, but was only partially inhibited by $\mathrm{CFTR}_{\text {inh }}-172$ or $\mathrm{CaCC}_{\text {inh }}-\mathrm{A} 01$ alone.

\section{Inhibition of Intestinal Motility by Kobusin and Eudesmin}

Since ANO1 is expressed in the pacemaker cells that generate smooth muscle contraction in the gastrointestinal tract (Huang et al., 2009; Hwang et al., 2009; Ferrera et al., 2010), more experiments were performed in vivo to evaluate the inhibitory effects of kobusin and eudesmin on gastrointestinal motility. Oral administration of either kobusin or eudesmin inhibited intestinal peristalsis and delayed charcoal movement in mice, 


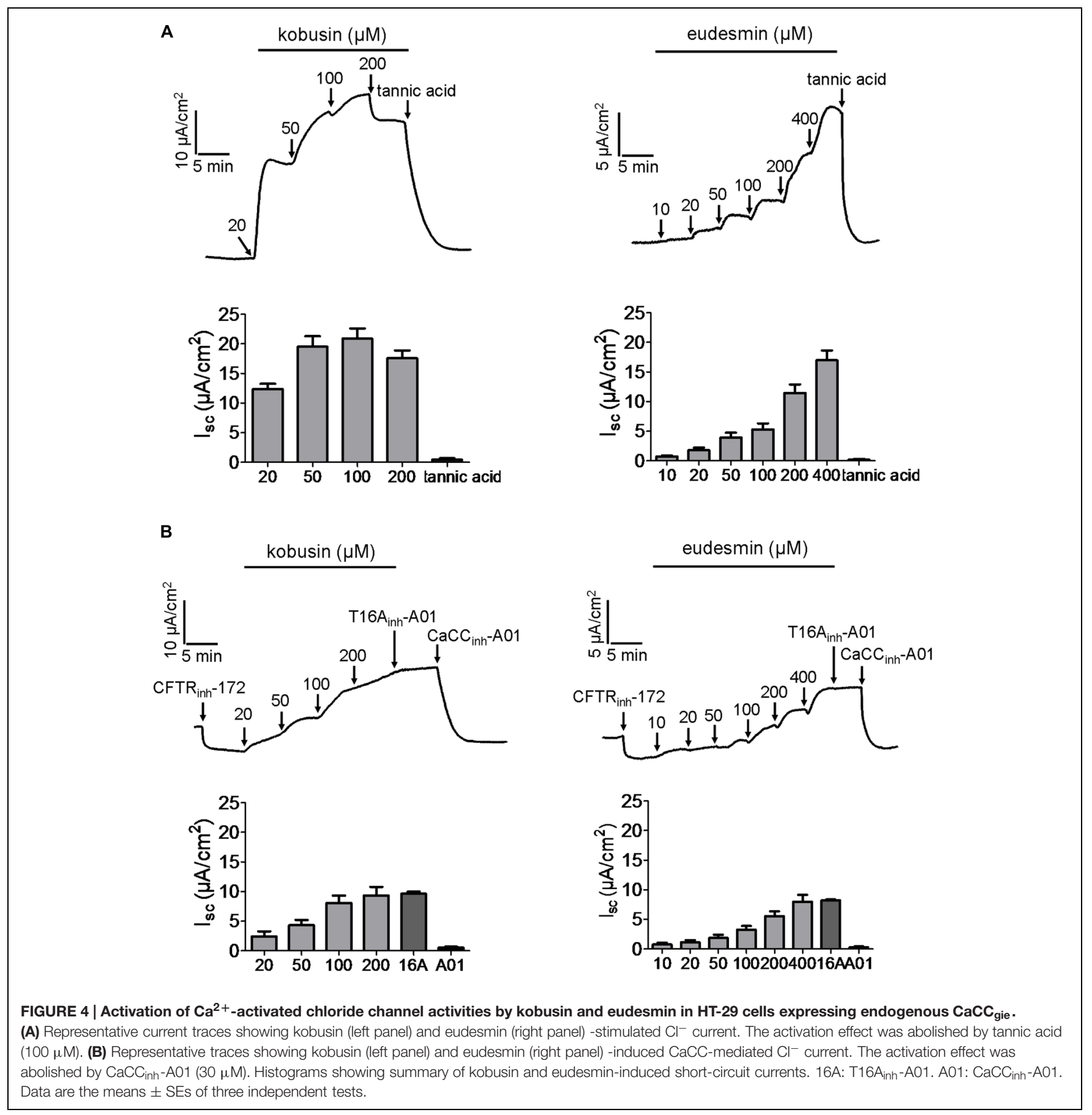

with peristaltic indexes of $70.6 \pm 5.9 \%$ in the case of kobusin and $68.2 \pm 5.9 \%$ in the case of eudesmin, compared to that of PBS $(82.3 \pm 2.9 \%$; Figures $8 \mathrm{~A}, \mathbf{B})$.

\section{DISCUSSION}

Lignans, described as a group of diphenolic compounds where the C6-C3 carbons are bound by the C8 central carbon, are widely distributed in more than 70 families of vascular plants. They have been isolated from different parts of a plant, including roots, stems, rhizomes, leaves, seeds, and fruits as well as the exudates and resins (Gang et al., 1997; Pan et al., 2009). Although neither non-nutrient nor noncaloric, lignans have attracted considerable attention because of their various biological activities. Numerous studies have shown that lignans and their intestinal metabolites enterolignans possess antitumor, antiviral, and antioxidant activities (Chen et al., 1997; Ashakumary et al., 1999; Saarinen et al., 2002). Furthermore, they have also been shown to have osteoporosis 
A

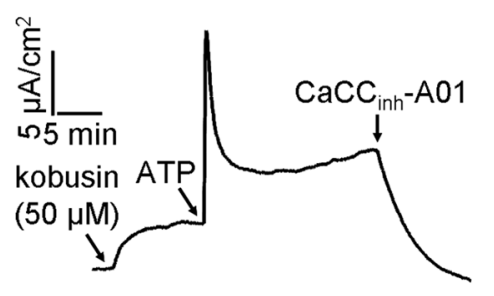

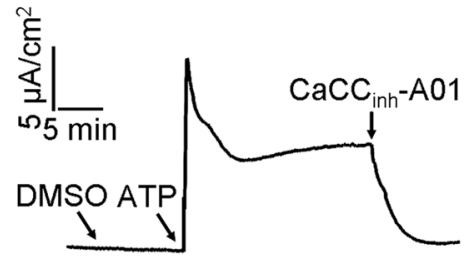

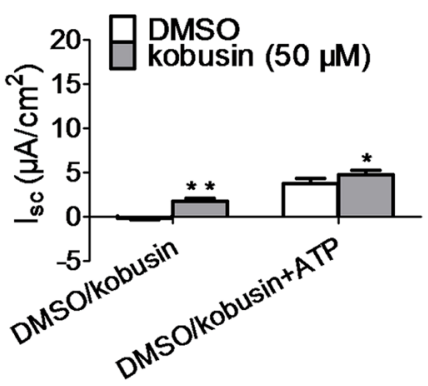

B
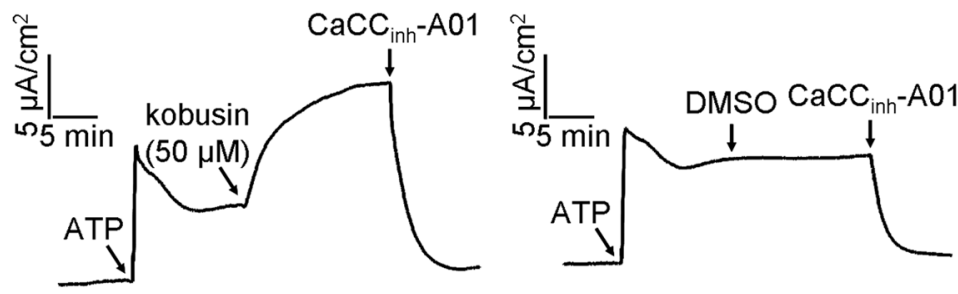

C
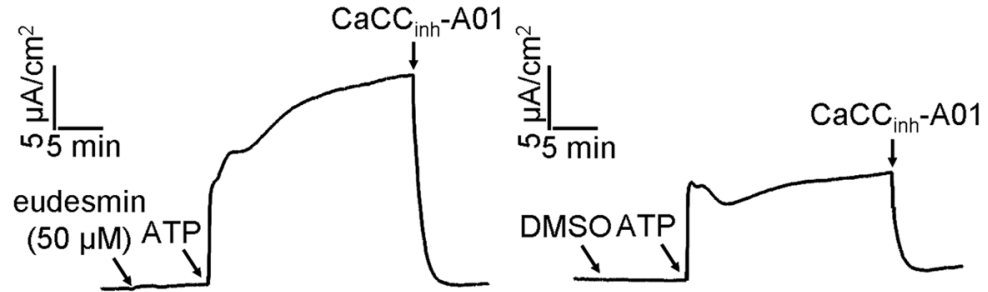

D

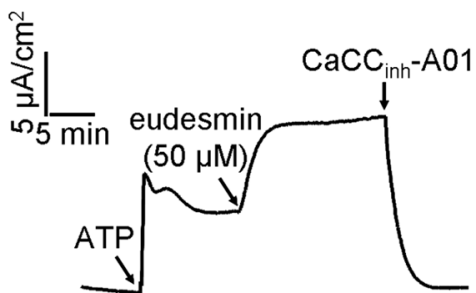

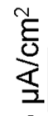

is $\mathrm{min}$

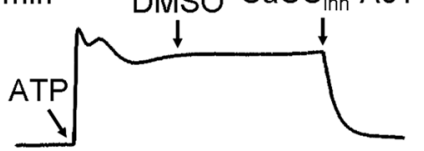

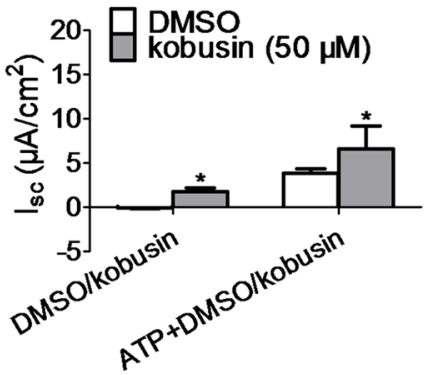
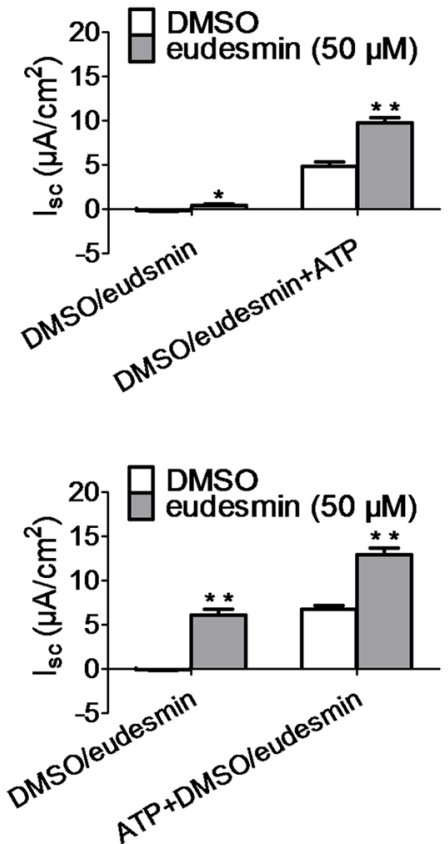

FIGURE 5 | Characteristic of $\mathbf{C a C C}_{\text {gie }}$ potentiation by kobusin and eudesmin in $\mathbf{H T}-29$ cells. (A,B) Representative traces of short-circuit currents showing synergistic effect between ATP and kobusin on the potentiation of $\mathrm{CaCC}_{\text {gie }}$ chloride channel. ATP was added after (A) and before (B) incubation with $50 \mu \mathrm{M}$ kobusin. (C,D) Representative traces of short-circuit currents showing synergistic effect between ATP and eudesmin on CaCC ${ }_{\text {gie }}$ chloride channel potentiation. ATP was added after (C) and before (D) incubation with the indicated $50 \mu \mathrm{M}$ eudesmin. CaCC $_{\text {gie }}$ Currents were abolished with CaCC $C_{\text {inh }}$-A01 at the end of tests.

Statistical data are shown in the right panel of (A-D). Data are the means \pm SEs of three independent tests. ${ }^{*} P<0.05,{ }^{* *} P<0.01$.

prevention and liver-protection activities as well as antagonistic activity toward platelet-activating factor (PAF; Han et al., 1992; Habauzit and Horcajada, 2008). In the present study, we demonstrated that two lignan compounds, kobusin and eudesmin, could function as activators of CFTR and $\mathrm{CaCC}_{\text {gie }}$ chloride channels and inhibitors of ANO1/CaCC channel. The results revealed that kobusin and eudesmin could activate the function of CFTR and $\mathrm{CaCC}_{\text {gie }}$ chloride channels. Notably, we 


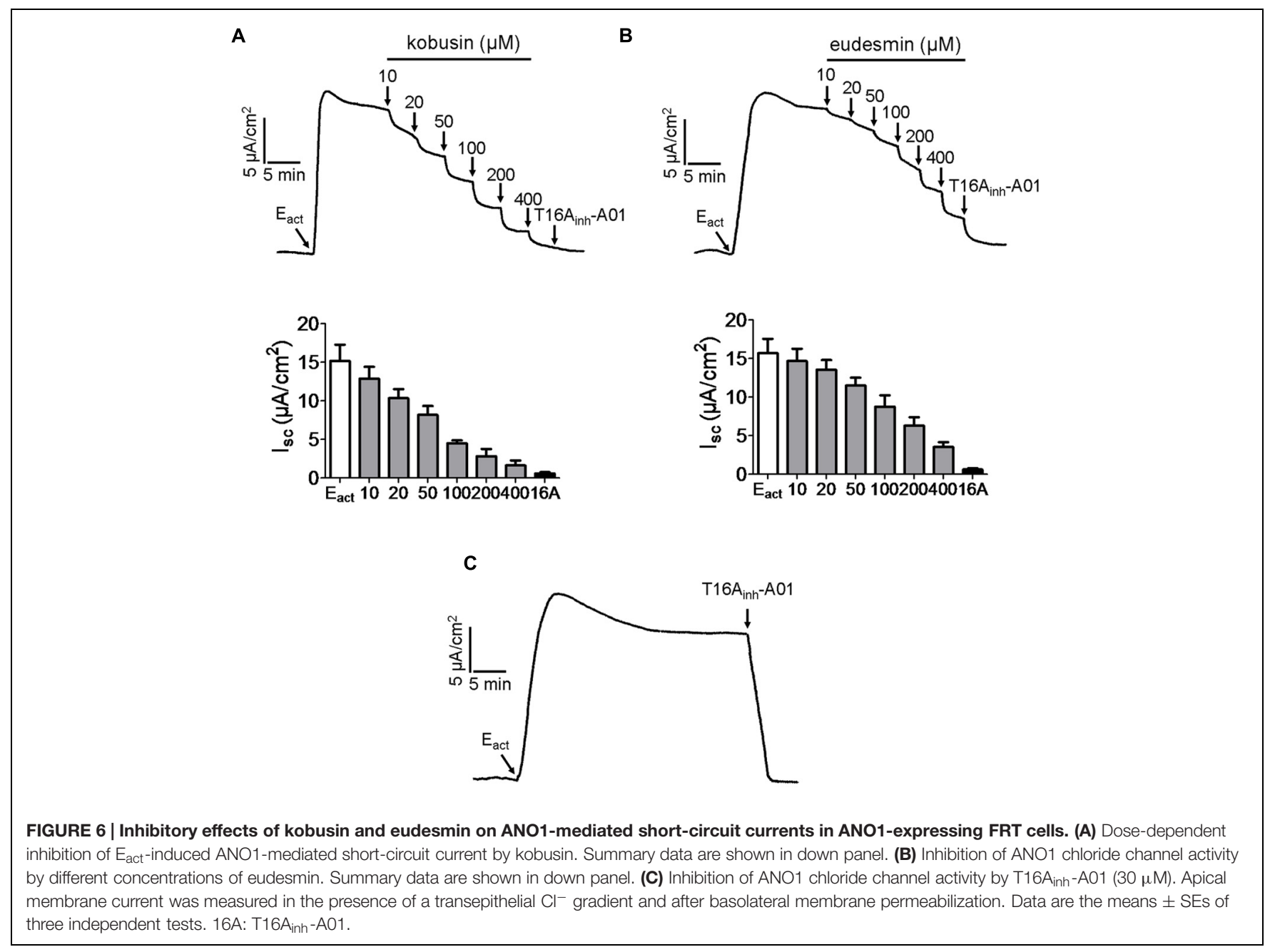

found that kobusin and eudesmin could inhibit the activities ANO1/CaCC chloride channel in ANO1/CaCC-expressing FRT cells, and reduce gastrointestinal motility in mice, thereby uncovering new molecular pharmacological targets of lignan compounds.

The identification of lignan compounds as CFTR and $\mathrm{CaCC}_{\text {gie }}$ activators would highlight their potential uses as lead drugs for the treatment of constipation. Intestinal fluid secretion provides a proper environment for digestion and facilitates stool passage through the intestinal tract (Barrett and Keely, 2000), and this process is driven by chloride channel-mediated $\mathrm{Cl}^{-}$transport in the enterocyte (Barrett and Keely, 2000; Kiela and Ghishan, 2009). So far, three chloride channels (namely CFTR, CaCC, and ClC-2) have been identified to mediate $\mathrm{Cl}^{-}$secretion into the intestinal lumen side, and among these, CFTR and CaCC play pivotal roles (Murek et al., 2010). In the intestine, CFTR, a cAMP-activated chloride channel, is mainly expressed in the crypt (Zhang et al., 2012). Mutation in the CFTR protein (e.g., $\triangle$ F508-CFTR) may result in the hereditary lethal disease of CF (Lubamba et al., 2012). Habitual constipation remains a common symptom among CF patients, which is regarded as a consequence of impaired intestinal fluid secretion (Grubb and Gabriel, 1997). Since the cloning of the CFTR gene back in 1989 (Riordan et al., 1989), CFTR has been advocated as a potential therapeutic molecular target for the treatment of several diseases, including CF (Kerem et al., 1989; Riordan et al., 1989), chronic pancreatitis (Cohn, 2005), habitual constipation (Morris et al., 1999), secretory diarrhea and autosomal dominant polycystic kidney disease (Li et al., 2004). Though the molecular identity of $\mathrm{CaCC}_{\text {gie }}$ still remains elusive, its existence in enterocyte has been fully confirmed (De La Fuente et al., 2008). $\mathrm{CaCC}_{\text {gie }}$ is responsible for rotaviral enterotoxin-stimulated diarrhea (Ko et al., 2014). In the present study, we demonstrated that kobusin and eudesmin could potentiate the two major pathways of $\mathrm{Cl}^{-}$secretion, suggesting that mild activation of CFTR and $\mathrm{CaCC}_{\text {gie }}$ may result in a significant activation of fluid secretion in the intestine. However, kobusin and eudesmin also inhibited the activity of ANO1/CaCC chloride channel (Figure 6). Kobusin and eudesmin inhibited ANO1/CaCCmediated short-circuit current in transfected FRT cells, and reduced gastrointestinal motility in mice. ANO1/CaCC is highly expressed in the pacemaker Cajal cells of the gastrointestinal tract (Huang et al., 2009; Hwang et al., 2009; Ferrera et al., 

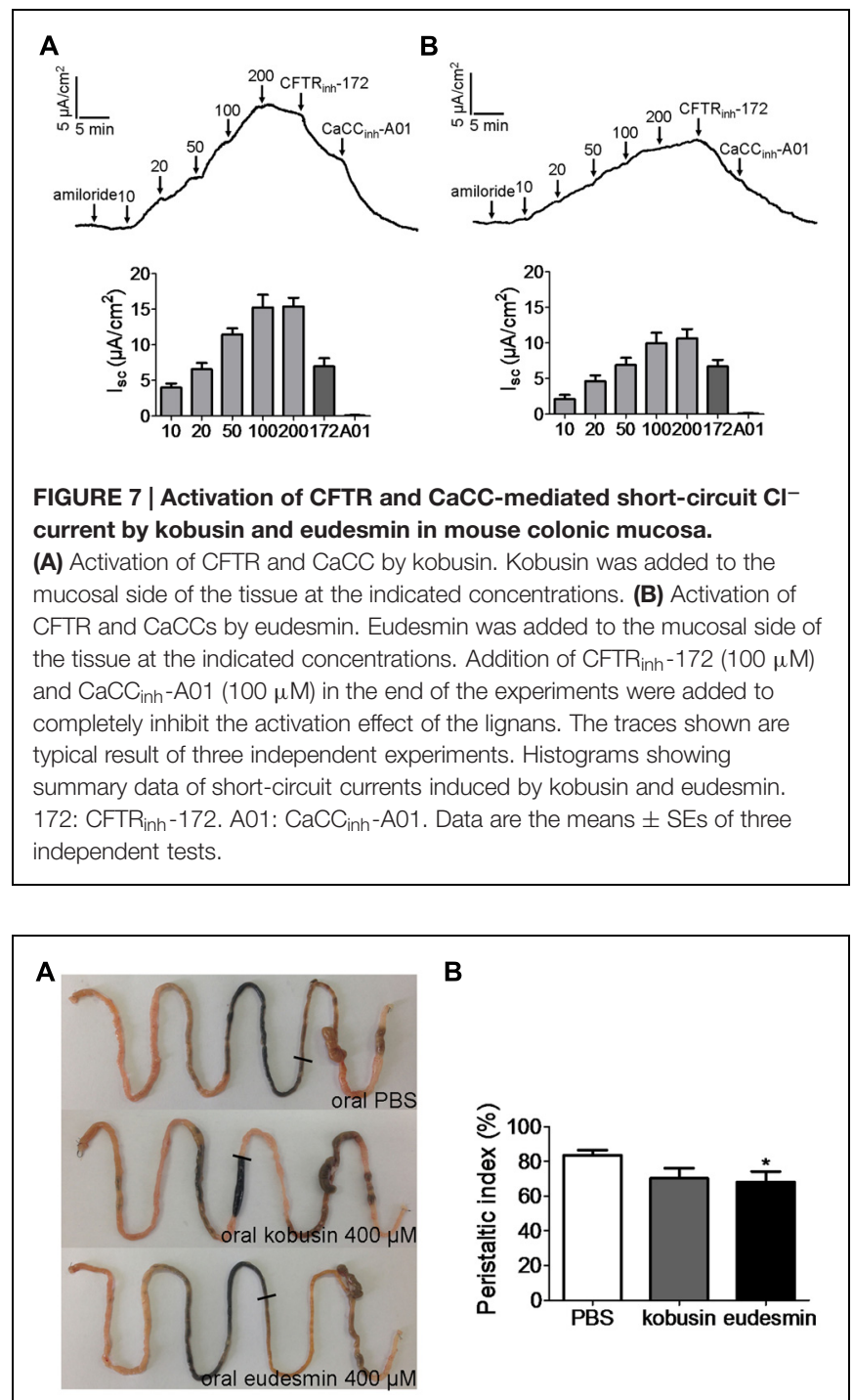

FIGURE 8 | Inhibition of gastrointestinal motility by kobusin and eudesmin in mice. (A) Photographs of isolated mouse intestinal tracts showing the distance traveled by activated charcoal after oral gavage of PBS (negative control) or kobusin and eudesmin. (B) Summary of the peristaltic indexes. Data are the means \pm SEs of three independent experiments. $* P<0.05$.

2010). Inhibition of ANO1/CaCC may delay the movement of the intestine and thus increase the fluid absorption time (Hwang et al., 2009). Thus the neutralization effect of these compounds on fluid secretion needs to be fully considered.

The study of lignan supplementation in some randomized controlled trials has indicated that lignans cause a mild but significant reduction in diastolic or/and systolic blood pressure in patients with hypertension (Ursoniu et al., 2015). Raimundo et al. (2009) reported that eudesmin can induce endothelium-dependent relaxation in rat aorta. The molecular mechanism of this effect still remains elusive. Accumulating evidence suggests that $\mathrm{ANO} / \mathrm{CaCC}$ plays important roles in the pathogenesis of spontaneous hypertension. Moreover, inhibition of ANO1/CaCC channel activity can reduce blood pressure in rodents so that spontaneous hypertension can be inhibited (Wang et al., 2015). The fact that kobusin and eudesmin could inhibit the activity of ANO1/CaCC channel both in vitro and in vivo suggested that inhibition of $\mathrm{ANO} / \mathrm{CaCC}$ may in part account for the antihypertension activities of the lignans.

Both epidemiological data and experimental evidence have indicated that lignans or lignan-rich food possess anticarcinogenic activities against many types of cancer, including breast (Saarinen et al., 2007), prostate (McCann et al., 2005), and colon (Webb and McCullough, 2005) cancers. The mechanisms involved in the cancer prevention effects are related to the anti-estrogenic, anti-angiogenic, pro-apoptotic, and antioxidant activities of these compounds (Webb and McCullough, 2005). Recently, it has been confirmed that $\mathrm{ANO} 1 / \mathrm{CaCC}$ is overexpressed in several tumors (Qu et al., 2014; Wanitchakool et al., 2014), and inhibition of ANO1/CaCC channel activity may suppress the proliferation and migration of cancer cells (Sui et al., 2014; Seo et al., 2015). The inhibition effect of kobusin and eudesmin on ANO1/CaCC channel activities observed in this study may provide new insight into the molecular mechanism associated with the anticancer effect of lignans.

The botanical properties of lignans have not been unveiled. Although prevalent in plants, lignans are virtually not found in animals (Peterson et al., 2010). The biosynthesis pathways of lignans are thought to have evolved in plants during their adaptation to the land (Davin and Lewis, 2000). Accumulating evidence shows that lignans are produced by phenoxy-radical coupling and polymerization, in which the dirigent proteins play key roles in determining the regiospecificity and stereoselectivity of the compounds (Gang et al., 1999), while the precise molecular mechanism is still unclear. In general, the lignan content in food is low except for flax seed, rye bran, and sesame seeds (Hallmans et al., 2003; Peterson et al., 2010). The demand for lignans has been increasing rapidly. The inefficiency and instability of plant lignan production means that there is an urgent need for a new technology to produce lignan. Recent studies have shed light on the production of lignans using transgenic plants and cells (Satake et al., 2013). Previous study has reported that ATP can activate $\mathrm{CaCC}$ chloride channel activities through both PLC and intracellular $\mathrm{Ca}^{2+}$ pathways (Rajagopal et al., 2011). We detected a synergistic effect between kobusin or eudesmin and ATP, which suggested that these compounds activated $\mathrm{CaCC}_{\text {gie }} \mathrm{Cl}^{-}$channel function in a way that may differ from ATP. Furthermore, we would like to know how these two lignan compounds inhibited ANO1 chloride channel function. Since $\mathrm{CaCCs}$ are $\mathrm{Ca}^{2+}$-activated chloride channels, the inhibition of $\mathrm{Ca}^{2+}$ release may impair the function of ANO1/CaCC or $\mathrm{CaCC}_{\text {gie }}$ channels. Zhang et al. (2013) reported that the lignan compound magnolol can inhibit colonic motility through down-regulating the voltage-sensitive L-type $\mathrm{Ca}^{2+}$ channel activities in rat colonic smooth muscle cells. Although both FRT and HT-29 cells express L-type calcium channels (Montiel et al., 2001; Perego et al., 2012), the adverse effect of 
kobusin and eudesmin on $\mathrm{CaCC}_{\text {gie }}$ and $\mathrm{ANO} 1$ chloride channel activities observed in our study did not support the L-type $\mathrm{Ca}^{2+}$ channel inhibition pathway. The detailed mechanisms will need further investigation.

\section{CONCLUSION}

The present study discovered the modulation of chloride channel function as a new activity of the lignan compounds kobusin and eudesmin, thereby uncovering new insights into the mechanism

\section{REFERENCES}

Ashakumary, L., Rouyer, I., Takahashi, Y., Ide, T., Fukuda, N., Aoyama, T., et al. (1999). Sesamin, a sesame lignan, is a potent inducer of hepatic fatty acid oxidation in the rat [J]. Metabolism 48, 1303-1313. doi: 10.1016/S00260495(99)90272-X

Barrett, K. E., and Keely, S. J. (2000). Chloride secretion by the intestinal epithelium: molecular basis and regulatory aspects. Annu. Rev. Physiol. 62, 535-572. doi: 10.1146/annurev.physiol.62.1.535

Chen, D. F., Zhang, S., Lan, X., Xie, J., Ke, C., Kashiwada, Y., et al. (1997). Anti-AIDS agents-XXVI. Structure-activity correlations of gomisin-G-related anti-HIV lignans from Kadsura interior and of related synthetic analogues [J]. Bioorg. Med. Chem. 5, 1715-1723. doi: 10.1016/S0968-0896(97)00118-1

Chen, L., Yu, B., Zhang, Y., Gao, X., Zhu, L., Ma, T., et al. (2015). Bioactivity-guided fractionation of an antidiarrheal Chinese herb Rhodiola kirilowii (Regel) Maxim reveals (-)-epicatechin-3-gallate and (-)-epigallocatechin-3-gallate as inhibitors of cystic fibrosis transmembrane conductance regulator. PLOS ONE 10:e0119122. doi: 10.1371/journal.pone.01 19122

Chun, J. N., Cho, M., So, I., and Jeon, J. H. (2014). The protective effects of Schisandra chinensis fruit extract and its lignans against cardiovascular disease: a review of the molecular mechanisms. Fitoterapia 97, 224-233. doi: 10.1016/j.fitote.2014.06.014

Clarke, L. L., Stien, X., and Walker, N. M. (2001). Intestinal bicarbonate secretion in cystic fibrosis mice. JOP 2(4 Suppl.), 263-267.

Cohn, J. A. (2005). Reduced CFTR function and the pathobiology of idiopathic pancreatitis [J]. J. Clin. Gastroenterol. 39, S70-S77. doi: 10.1097/01.mcg.0000155522.89005.bf

Dar, A. A., and Arumugam, N. (2013). Lignans of sesame: purification methods, biological activities and biosynthesis-a review. Bioorg. Chem. 50, 1-10. doi: 10.1016/j.bioorg.2013.06.009

Davin, L. B., and Lewis, N. G. (2000). Dirigent proteins and dirigent sites explain the mystery of specificity of radical precursor coupling in lignan andlignin biosynthesis. Plant Physiol. 123, 453-462. doi: 10.1104/pp.1 23.2.453

De La Fuente, R., Namkung, W., Mills, A., and Verkman, A. S. (2008). Smallmolecule screen identifies inhibitors of a human intestinal calcium-activated chloride channel. Mol. Pharmacol. 73, 758-768.

Ferrera, L., Caputo, A., and Galietta, L. J. (2010). TMEM16A protein: a new identity for $\mathrm{Ca}^{2+}$-dependent $\mathrm{Cl}^{-}$channels. Physiology 25, 357-363. doi: 10.1152/physiol.00030.2010

Gang, D. R., Costa, M. A., Fujita, M., Dinkova-Kostova, A. T., Wang, H. B., Burlat, V., et al. (1999). Regiochemical control of monolignol radical coupling: a new paradigm for lignin and lignan biosynthesis. Chem. Biol. 6, 143-151. doi: 10.1016/S1074-5521(99)89006-1

Gang, D., Dinkova-Kostova, A., Davin, L., and Lewis, N. (1997). "Phylogenetic links in plant defense systems: lignans, isoflavonoids, and their reductases," in Phytochemicals for Pest Control, eds. P. A. Hedin, R. M. Hollingworth, E. P. Masler, and J. Miyamoto (Washington, DC: American Chemical Society), 59-89.

Garcia, M. A., Yang, N., and Quinton, P. M. (2009). Normal mouse intestinal mucus release requires cystic fibrosis transmembrane regulator-dependent bicarbonate secretion. J. Clin. Invest. 119, 2613-2622. doi: 10.1172/JCI38662 relating to the antihypertension and cancer prevention activities of lignans in general.

\section{ACKNOWLEDGMENT}

This work was supported by National Natural Science Fund (No. 31471099; 81173109; 81473265), Special Fund for Doctorate Disciplines Construction in Universities (20112136110002) and the Youth Foundation of Liaoning Normal University (No. LS2014L010).

Grubb, B. R., and Gabriel, S. E. (1997). Intestinal physiology and pathology in gene-targeted mouse models of cystic fibrosis. Am. J. Physiol. 273, G258-G266.

Habauzit, V., and Horcajada, M. N. (2008). Phenolic phytochemicals and bone. Phytochem. Rev. 7, 313-344. doi: 10.1007/s11101-007-9078-9

Hallmans, G., Zhang, J. X., Lundin, E., Stattin, P., Johansson, A., Johansson, I., et al. (2003). Rye, lignans and human health. Proc. Nutr. Soc. 62, 193-199. doi: 10.1079/PNS2002229

Han, G., Dai, P., Xue, R., Arison, B. H., Lankin, D. C., and Hwang, S. (1992). Dibenzocyclooctadiene Lignans with platelet-activating factor (PAF) antagonist activity from Kadsura heteroclita [J]. J. Chin. Pharmaceut. Sci. 1:20.

Hao, F., Yi, F., Zhang, D., Ning, Y., Su, W., Feng, X., et al. (2011). Identification of herbal compound imperatorin with adverse effects on ANO1 and CFTR chloride channels. Chem. Res. Chinese Univ. 27, 461-463.

Harmon, G. S., Dumlao, D. S., Ng, D. T., Barrett, K. E., Dennis, E. A., Dong, H., et al. (2010). Pharmacological correction of a defect in PPAR-gamma signaling ameliorates disease severity in Cftr-deficient mice. Nat. Med. 16, 313-318. doi: 10.1038/nm.2101

Hartzell, C., Putzier, I., and Arreola, J. (2005). Calcium-activated chloride channels. Annu. Rev Physiol. 67, 719-758. doi: 10.1146/annurev.physiol.67.032003.154341

Huang, F., Rock, J. R., Harfe, B. D., Cheng, T., Huang, X., Jan, Y. N., et al. (2009). Studies on expression and function of the TMEM16A calciumactivated chloride channel. Proc. Natl. Acad. Sci. U.S.A. 106, 21413-21418. doi: 10.1073/pnas.0911935106

Hwang, S. J., Blair, P. J., Britton, F. C., O’Driscoll, K. E., Hennig, G., Bayguinov, Y. R., et al. (2009). Expression of anoctamin 1/TMEM16A by interstitial cells of Cajal is fundamental for slow wave activity in gastrointestinal muscles. J. Physiol. 587, 4887-4904. doi: 10.1113/jphysiol.2009. 176198

Hwang, T. C., and Sheppard, D. N. (1999). Molecular pharmacology of the CFTR $\mathrm{Cl}^{-}$channel. Trends Pharmacol. 20, 448-453. doi: 10.1016/S01656147(99)01386-3

Hwang, T. C., Wang, F., Yang, I. C., and Reenstra, W. W. (1997). Genistein potentiates wild-type and delta F508-CFTR channel activity. Am. J. Physiol. 273, C988-C998.

Kerem, B., Rommens, K. M., Buchanan, D., Mardiewicz, D., Cox, T. K., Chakravarti, A., et al. (1989). Identification of the cystic gene, genetic analysis. Science 245, 1073-1080. doi: 10.1126/science.2570460

Kiela, P. R., and Ghishan, F. K. (2009). Ion transport in the intestine. Curr. Opin. Gastroenterol. 25, 87-91. doi: 10.1097/MOG.0b013e32832 60900

Ko, E. A., Jin, B. J., Namkung, W., Ma, T., Thiagarajah, J. R., and Verkman, A. S. (2014). Chloride channel inhibition by a red wine extract and a synthetic small molecule prevents rotaviral secretory diarrhoea in neonatal mice. Gut 63, 1120-1129. doi: 10.1136/gutjnl-2013-305663

Kristidis, P., Bozon, D., Corey, M., Markiewicz, D., Rommens, J., Tsui, L. C., et al. (1992). Genetic determination of exocrine pancreatic function in cystic fibrosis. Am. J. Hum. Genet. 50, 1178-1184.

Lembo, A., and Camilleri, M. (2003). Chronic constipation. N. Engl. J. Med. 349, 1360-1368. doi: 10.1056/NEJMra020995

Li, H., Findlay, I. A., and Sheppard, D. N. (2004). The relationship between cell proliferation, $\mathrm{Cl}^{-}$secretion, and renal cyst growth: a study using CFTR inhibitors [J]. Kidney Int. 66, 1926-1938. 
Lu, M., Yang, B., Liu, Y., Liu, Q., and Wen, H. (2015). Procedure for prolapse and hemorrhoids vs traditional surgery for outlet obstructive constipation. World J. Gastroenterol. 21, 8178-8183. doi: 10.3748/wjg.v21.i26.8178

Lubamba, B., Dhooghe, B., Noel, S., and Leal, T. (2012). Cystic fibrosis: insight into CFTR pathophysiology and pharmacotherapy. Clin. Biochem. 45, 1132-1144. doi: 10.1016/j.clinbiochem.2012.05.034

Ma, T., Vetrivel, L., Yang, H., Pedemonte, N., Zegarra-Moran, O., Galietta, L. J., et al. (2002). High-affinity activators of cystic fibrosis transmembrane conductance regulator (CFTR) chloride conductance identified by high-throughput screening. J. Biol. Chem. 277, 37235-37241. doi: 10.1074/jbc.M205932200

McCann, M. J., Gill, C. I., McGlynn, H., and Rowland, I. R. (2005). Role of mammalian lignans in the prevention and treatment of prostate cancer. Nutr. Cancer 52, 1-14. doi: 10.1207/s15327914nc5201_1

Montiel, M., Pavía, J., Marsigliante, S., and Jiménez, E. (2001). Activation of muscarinic acetylcholine receptors induces $\mathrm{Ca}^{2+}$ mobilization in FRT cells. Cell. Signal. 13, 207-212. doi: 10.1016/S0898-6568(01)00133-4

Morris, A. P., and Frizzell, R. A. (1993). Ca(2+)-dependent Cl- channels in undifferentiated human colonic cells (HT-29). I. Single-channel properties. Am. J. Physiol. 264(4 Pt 1), C968-C976.

Morris, A. P., Scott, J. K., Ball, J. M., Zeng, C., O'Neal, W. K., and Estes, M. K. (1999). NSP4 elicits age-dependent diarrhea and $\mathrm{Ca}^{2+}$ mediated $\mathrm{I}^{-}$influx into intestinal crypts of CF mice. Am. J. Physiol. 277, G431-G444.

Murek, M., Kopic, S., and Geibel, J. (2010). Evidence for intestinal chloride secretion. Exp. Physiol. 95, 471-478. doi: 10.1113/expphysiol.2009.049445

Namkung, W., Phuan, P. W., and Verkman, A. S. (2011a). TMEM16A inhibitors reveal TMEM16A as a minor component of calciumactivated chloride channel conductance in airway and intestinal epithelial cells. J. Biol. Chem. 286, 2365-2374. doi: 10.1074/jbc.M110.1 75109

Namkung, W., Yao, Z., Finkbeiner, W. E., and Verkman, A. S. (2011b). Smallmolecule activators of TMEM16A, a calcium-activated chloride channel, stimulate epithelial chloride secretion and intestinal contraction. FASEB J. 25, 4048-4062. doi: 10.1096/fj.11-191627

Pan, J., Chen, S., Yang, M., Wu, J., Sinkkonen, J., and Zou, K. (2009). An update on lignans: natural products and synthesis. Nat. Prod. Rep. 26, 1251-1292. doi: 10.1039/b910940d

Perego, S., Cosentino, S., Fiorilli, A., Tettamanti, G., and Ferraretto, A. (2012). Casein phosphopeptides modulate proliferation, and apoptosis in HT-29 cell line through their interaction with voltage-operated L-type calcium channels. J. Nutr. Biochem. 23, 808-816. doi: 10.1016/j.jnutbio.2011.04.004

Peterson, J., Dwyer, J., Adlercreutz, H., Scalbert, A., Jacques, P., and McCullough, M. L. (2010). Dietary lignans: physiology and potential for cardiovascular disease risk reduction. Nutr. Rev. 68, 571-603. doi: 10.1111/j.1753-4887.2010.00319.x

Qu, Z., Yao, W., Yao, R., Liu, X., Yu, K., and Hartzell, C. (2014). The $\mathrm{Ca}(2+)$-activated $\mathrm{Cl}(-)$ channel, ANO1 (TMEM16A), is a double-edged sword in cell proliferation and tumorigenesis. Cancer Med. 3, 453-461. doi: $10.1002 / \mathrm{cam} 4.232$

Raimundo, J. M., Trindade, A. P., Velozo, L. S., Kaplan, M. A., Sudo, R. T., and Zapata-Sudo, G. (2009). The lignan eudesmin extracted from Piper truncatum induced vascular relaxation via activation of endothelial histamine H1 receptors. Eur. J. Pharmacol. 606, 150-154. doi: 10.1016/j.ejphar.2009.0 1.038

Rajagopal, M., Kathpalia, P. P., Thomas, S. V., and Pao, A. C. (2011). Activation of $\mathrm{P} 2 \mathrm{Y} 1$ and $\mathrm{P} 2 \mathrm{Y} 2$ receptors induces chloride secretion via calcium-activated chloride channels in kidney inner medullary collecting duct cells. Am. J. Physiol. Renal Physiol. 301, F544-F553. doi: 10.1152/ajprenal.00709.2010

Riordan, J. R., Rommens, J. M., Kerem, B., Alon, N., Rozmahel, R., Grzelczak, Z., et al. (1989). Identification of the cystic fibrosis gene: cloning and characterization of complementary DNA. Science 245, 1437. doi: $10.1126 /$ science. 2475911
Saarinen, N. M., Smeds, A., Mäkelä, S. I., Ammälä, J., Hakala, K., Pihlava, J. M., et al. (2002). Structural determinants of plant lignans for the formation of enterolactone in vivo. J. Chromatogr. B Analyt. Technol. Biomed. Life Sci. 777, 311-319. doi: 10.1016/S1570-0232(02)00339-2

Saarinen, N. M., Wärri, A., Airio, M., Smeds, A., and Mäkelä, S. (2007). Role of dietary lignans in the reduction of breast cancer risk. Mol. Nutr. Food Res. 51, 857-866. doi: 10.1002/mnfr.200600240

Satake, H., Ono, E., and Murata, J. (2013). Recent advances in the metabolic engineering of lignan biosynthesis pathways for the production of transgenic plant-based foods and supplements. J. Agric. Food Chem. 61, 11721-11729. doi: $10.1021 /$ jf 4007104

Schultz, B. D., Singh, A. K., Devor, D. C., and Bridges, R. J. (1999). Pharmacology of CFTR chloride channel activity. Physiol. Rev. 79(1 Suppl.), S109-S144.

Seo, Y., Park, J., Kim, M., Lee, H. K., Kim, J. H., Jeong, J. H., et al. (2015). Inhibition of ANO1/TMEM16A chloride channel by idebenone and its cytotoxicity to cancer cell lines. PLOS ONE 10:e0133656. doi: 10.1371/journal.pone. 0133656

Sheppard, D. N., and Welsh, M. J. (1999). Structure and function of the CFTR chloride channel. Physiol. Rev. 79(1 Suppl.), S23-S45.

Sui, Y., Sun, M., Wu, F., Yang, L., Di, W., Zhang, G., et al. (2014). Inhibition of TMEM16A expression suppresses growth and invasion in human colorectal cancer cells. PLoS ONE 9:e115443. doi: 10.1371/journal.pone.0115443

Ursoniu, S., Sahebkar, A., Andrica, F., Serban, C., Banach, M., and Lipid, and Blood Pressure Meta-analysis Collaboration, LBPMC Group, (2015). Effects of flaxseed supplements on blood pressure: a systematic review and meta-analysis of controlled clinical trial. Clin. Nutr. doi: 10.1016/j.clnu.2015.05.012 [Epub ahead of print].

Wang, B., Li, C., Huai, R., and Qu, Z. (2015). Overexpression of ANO1/TMEM16A, an arterial $\mathrm{Ca}^{2+}$-activated $\mathrm{Cl}^{-}$channel, contributes to spontaneous hypertension. J. Mol. Cell Cardiol. 82, 22-32. doi: 10.1016/j.yjmcc.2015.02.020

Wanitchakool, P., Wolf, L., Koehl, G. E., Sirianant, L., Schreiber, R., Kulkarni, S., et al. (2014). Role of anoctamins in cancer and apoptosis. Philos. Trans. R. Soc. Lond. B Biol. Sci. 369, 20130096. doi: 10.1098/rstb.2013.0096

Webb, A. L., and McCullough, M. L. (2005). Dietary lignans: potential role in cancer prevention. Nutr. Cancer 51, 117-131. doi: 10.1207/s15327914nc 5102_1

Zhang, J., Chen, J., Liang, Z., and Zhao, C. (2014a). New lignans and their biological activities. Chem. Biodivers. 11, 1-54. doi: 10.1002/cbdv.201100433

Zhang, Y., Yu, B., Sui, Y., Gao, X., Yang, H., and Ma, T. (2014b). Identification of resveratrol oligomers as inhibitors of cystic fibrosis transmembrane conductance regulator by high-throughput screening of natural products from chinese medicinal plants. PLoS ONE 9:e94302. doi: 10.1371/journal.pone.0094302

Zhang, M., Zang, K., Luo, J., Leung, F., Huang, Y., Lin, C., et al. (2013). Magnolol inhibits colonic motility through down-regulation of voltage-sensitive L-type $\mathrm{Ca}^{2+}$ channels of colonic smooth muscle cells in rats. Phytomedicine 20, 1272-1279. doi: 10.1016/j.phymed.2013.07.008

Zhang, W., Fujii, N., and Naren, A. P. (2012). Recent advances and new perspectives in targeting CFTR for therapy of cystic fibrosis and enterotoxin-induced secretory diarrheas. Future Med. Chem. 4, 329-345. doi: $10.4155 /$ fmc. 12.1

Conflict of Interest Statement: The authors declare that the research was conducted in the absence of any commercial or financial relationships that could be construed as a potential conflict of interest.

Copyright $\odot 2015$ Jiang, Yu, Fang, Cao, Ma and Yang. This is an open-access article distributed under the terms of the Creative Commons Attribution License (CC BY). The use, distribution or reproduction in other forums is permitted, provided the original author(s) or licensor are credited and that the original publication in this journal is cited, in accordance with accepted academic practice. No use, distribution or reproduction is permitted which does not comply with these terms. 